\title{
Temperature-Dependent Characterization of SiC Power Electronic Devices
}

\author{
Madhu Sudhan Chinthavali ${ }^{1}$ \\ chinthavalim@ornl.gov \\ Burak Ozpineci $^{2}$ \\ burak@ieee.org \\ Leon M. Tolbert ${ }^{2,3}$ \\ tolbert@utk.edu \\ ${ }^{1}$ Oak Ridge Institute for Science and Education, Oak Ridge, TN 37831-0117 USA \\ ${ }^{2}$ Oak Ridge National Laboratory, Oak Ridge, TN 37831-6472 USA \\ ${ }^{3}$ The University of Tennessee, Department of Electrical and Computer Engineering, Knoxville, TN 37996-2100 USA
}

\begin{abstract}
Silicon (Si) unipolar devices are limited in breakdown voltages because of the low electric field strength of the material. Silicon carbide ( $\mathrm{SiC}$ ) unipolar devices, on the other hand, have 10 times greater electric field strength and hence they have much higher breakdown voltages compared with $\mathrm{Si}$. They also have low static and dynamic losses compared with Si devices. Four commercially available SiC Schottky diodes at different voltage and current ratings and an experimental SiC VJFET sample have been tested to characterize their performance at different temperatures. Their forward characteristics and switching characteristics in a temperature range of $-50^{\circ} \mathrm{C}$ to $175^{\circ} \mathrm{C}$ are presented. The results for the SiC Schottky diodes are compared with the results for a Si pn diode with comparable ratings. The experimental data were analyzed to obtain the device performance parameters like the on-state resistance and the switching losses.
\end{abstract}

\section{INTRODUCTION}

There is a growing demand for more efficient, higher power and higher temperature operation in the transportation industry. In spite of the advanced technology, silicon ( $\mathrm{Si}$ ) power devices have been saturated in terms of higher temperature and higher power operation by virtue of their physical properties. Silicon carbide (SiC) has been identified as a material with the potential to replace Si devices due to their superior material advantages such as large bandgap, high thermal conductivity, and high critical breakdown field strength. SiC devices are capable of operating at high voltages, high frequencies, and at higher junction temperatures. Significant reduction in weight and size of SiC power converters with an increase in the efficiency is projected [1-4].

SiC unipolar devices such as Schottky diodes, VJFETs, MOSFETs, etc. have much higher breakdown voltages compared to their Si counterparts which makes them suitable for use in traction drives. The power devices in traction applications should be able to handle extreme environments

Prepared by the Oak Ridge National Laboratory, Oak Ridge, Tennessee 37831, managed by UT-Battelle for the U.S. Department of Energy under contract DE-AC05-00OR22725.

The submitted manuscript has been authored by a contractor of the U.S. Government under Contract No. DE-AC05-00OR22725. Accordingly, the U.S. Government retains a non-exclusive, royalty-free license to publish from the contribution, or allow others to do so, for U.S. Government purposes. which include a wide range of operating temperature. In the following sections, the performance of some commercially available SiC Schottky diodes and experimental samples of $\mathrm{SiC}$ VJFETs will be presented.

\section{SiC SCHOTTKY DIODES}

SiC Schottky diodes are majority carrier devices and are attractive for high frequency applications because they have lower switching losses compared to pn diodes. However, Schottky diodes have higher leakage currents, which affect the breakdown voltage rating of the device [5]. A list of SiC Schottky diodes tested are S1 (1200V, 7.5A), S2 (600V, 4A), S3 (600 V, $10 \mathrm{~A})$, and S4 (300 V, 10A).

\section{A. Static Characteristics}

The static characteristics of different SiC Schottky diodes at room temperature are shown in Fig. 1. The threshold voltage or the knee voltage and the on-state resistance is different for the diodes because of differences in device dimensions for different ratings. The static characteristics of diodes for a temperature range of $-50^{\circ} \mathrm{C}$ to $175^{\circ} \mathrm{C}$ are shown in Fig. 2 to Fig. 6. The on-state voltage drop of a Schottky diode is dependent on barrier height and the on-state resistance. Both parameters vary with temperature and hence contribute to the temperature dependence of forward characteristics.

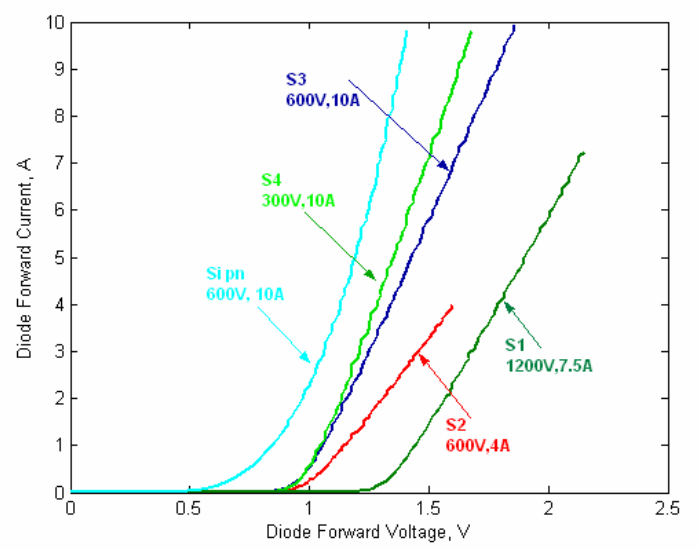

Fig. 1. $i-v$ characteristics of Si pn and SiC Schottky diodes at $27^{\circ} \mathrm{C}$. 


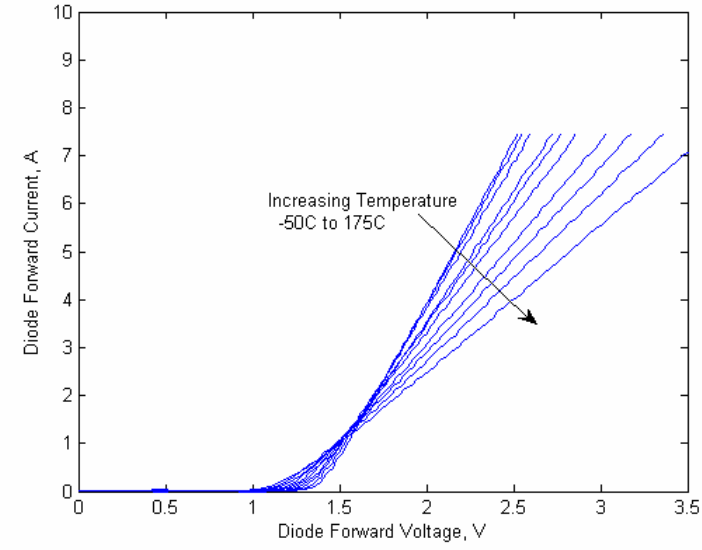

Fig. 2. $i-v$ characteristics of $S 1(1200 \mathrm{~V}, 7.5 \mathrm{~A})$ at different operating temperatures.

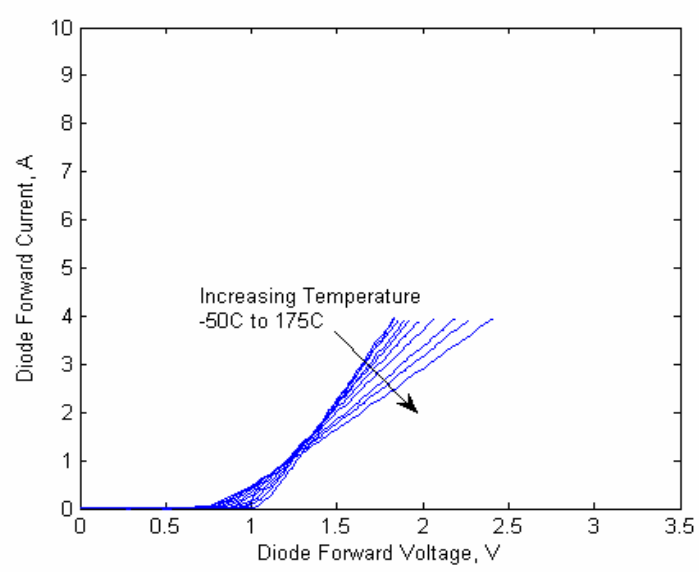

Fig. 3. $i-v$ characteristics of $\mathrm{S} 2(600 \mathrm{~V}, 4 \mathrm{~A})$ at different operating temperatures.

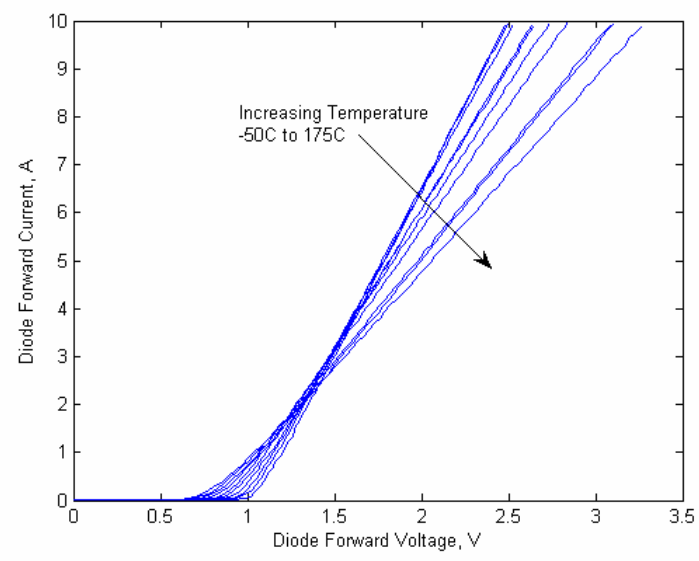

Fig. 4. $i-v$ characteristics of S3 $(600 \mathrm{~V}, 10 \mathrm{~A})$ at different operating temperatures.

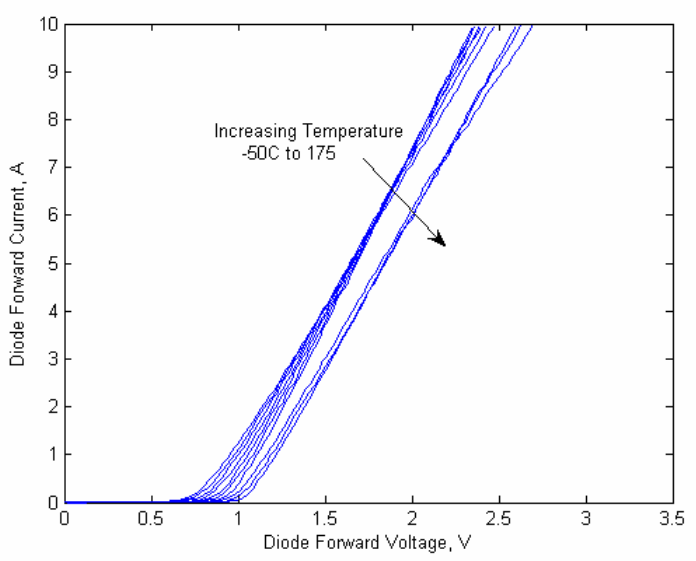

Fig. 5. $i-v$ characteristics of $S 4(300 \mathrm{~V}, 10 \mathrm{~A})$ at different operating temperatures.

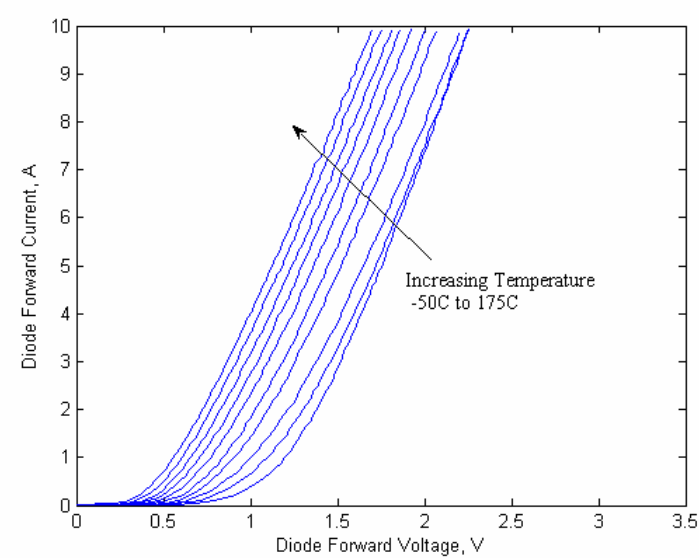

Fig. 6. $i-v$ characteristics of Si p-n diode $(600 \mathrm{~V}, 10 \mathrm{~A})$ at different operating temperatures.

At lower current levels the built-in potential (barrier potential) decreases with increasing temperature due to reduction in barrier height [6]. As the temperature increases, the thermal energy of electrons increase which causes lowering of the barrier height. The on-state voltage drop of the diode is given as,

$$
V_{f}=V_{d}+I_{d} \cdot R_{d}
$$

where $V_{d}$ is the forward voltage drop and $R_{d}$ is the series resistance of the diode obtained from the piece-wise linear (PWL) model of the diode. The PWL model parameters were extracted from the experimental data. The variation in $V_{d}$ with temperature is plotted in Fig. 7.

At higher current levels the voltage drop is mainly due to the series resistance of the diode [10]. The on-state resistance is one of the critical parameters, which determines the performance of the device and is a temperature sensitive 


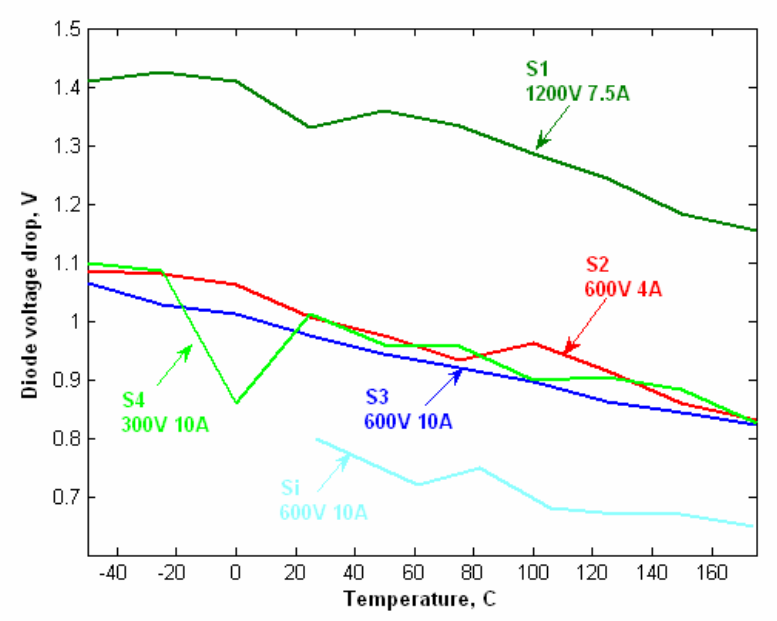

Fig. 7. $V_{d}$ for $\mathrm{Si}$ and $\mathrm{SiC}$ diodes at different operating temperatures.

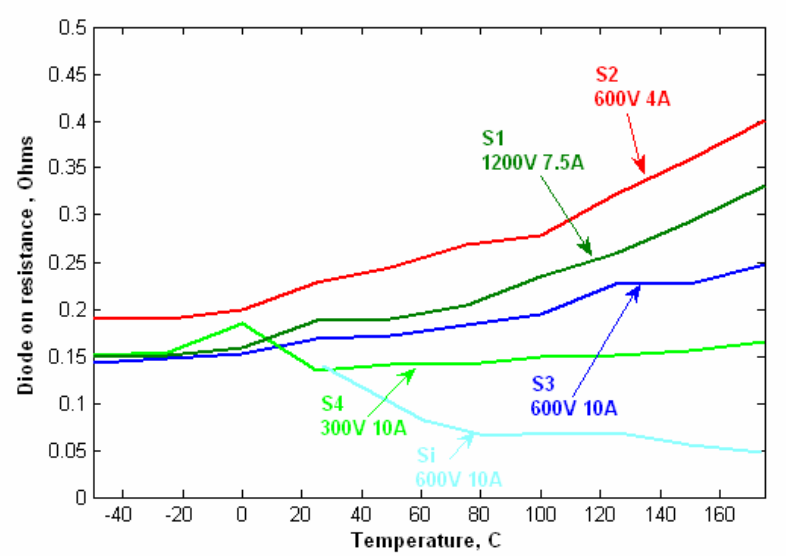

Fig. 8. $R_{d}$ for $\mathrm{Si}$ and $\mathrm{SiC}$ diodes at different operating temperatures.

parameter. $R_{o n, s p}$ increases with temperature due to decrease in mobility at higher temperatures. The positive temperature coefficient characteristic increases the conduction losses at high temperatures; however, this is advantageous for current sharing and paralleling.

The $R_{d}$ for the diodes was calculated from the slope of the $i-v$ characteristics and is plotted for different temperatures as shown in Fig. 8. The on-state resistance varies for each diode due to the difference in blocking voltages. The $R_{o n, s p}$ for majority carrier devices can be expressed as a function of breakdown voltage and critical electric field.

$$
R_{o n, s p}=\frac{4 V_{B}^{2}}{\varepsilon\left(E_{c}\right)^{3} \mu_{n}}
$$

where $\varepsilon$ is the permittivity $(\mathrm{C} / \mathrm{V} \cdot \mathrm{cm}), V_{B}$ is the breakdown voltage, $\mathrm{E}_{\mathrm{c}}$ is the breakdown field $(\mathrm{V} / \mathrm{cm})$, and $\mu_{n}$ is the electron mobility $\left(\mathrm{cm}^{2} / \mathrm{V} \cdot \mathrm{s}\right)$. To withstand high breakdown voltages, the blocking layer thickness is increased, and

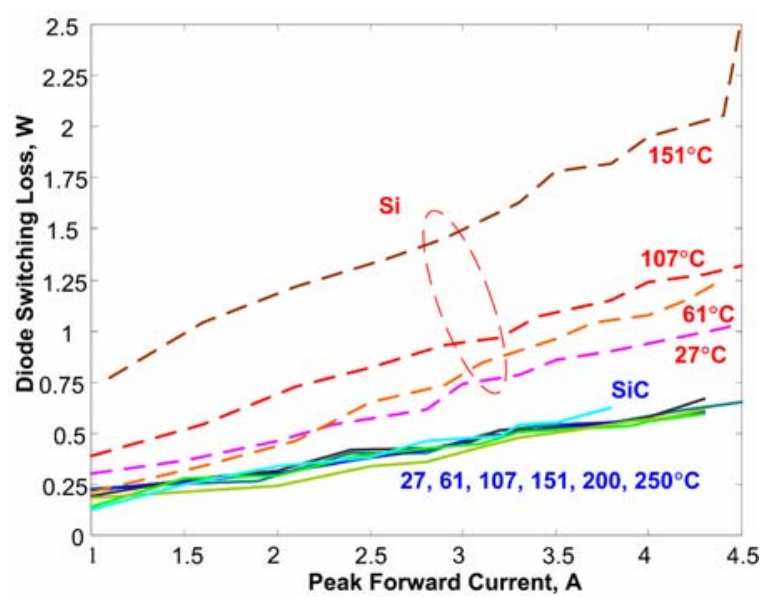

Fig. 9. Peak reverse recovery values with respect to forward current at different operating temperatures.

doping concentrations are reduced. This results in increased series resistance of the diode. Hence, device S1 rated at $1200 \mathrm{~V}, 7.5 \mathrm{~A}$ has more on-resistance compared to S3 and S4. The resistance varies with forward current density and the area of the device. It is evident from Fig. 8 that S2 and S3 with the same voltage and different current ratings have different on-state resistances.

\section{B. Dynamic Characteristics}

A buck chopper with an inductive load is built for evaluating the switching characteristics of the diodes. An IGBT is used as the main switch and is switched at $20 \mathrm{kHz}$ with a $25 \%$ duty ratio.

The power losses for various forward peak currents and different temperatures are shown for the Si diode and diode S4 in Fig. 9. The power losses for the Si diode increase with temperature, because of the increase in peak reverse recovery current. The switching loss for diode S4 is almost independent of the change in temperature. The reverse recovery current is dependent on charge stored in the drift region. The SiC Schottky diode has no stored charge because it is a majority carrier device, and hence has virtually constant turn on energy loss for a wide temperature range.

The negligible reverse recovery current reduces the oscillation due to ringing and also eliminates the need for a snubber for limiting the reverse recovery. This results in increased efficiency as the losses are minimized. Also, the reduced blocking layer thickness, due to the high electric breakdown field of the SiC material, contributes to the low switching losses of the SiC diode.

\section{SiC VERTICAL JFET (VJFET)}

\section{A. Static characteristics}

JFET is a unipolar device and has several advantages compared to MOSFET devices. JFET has low voltage drop 


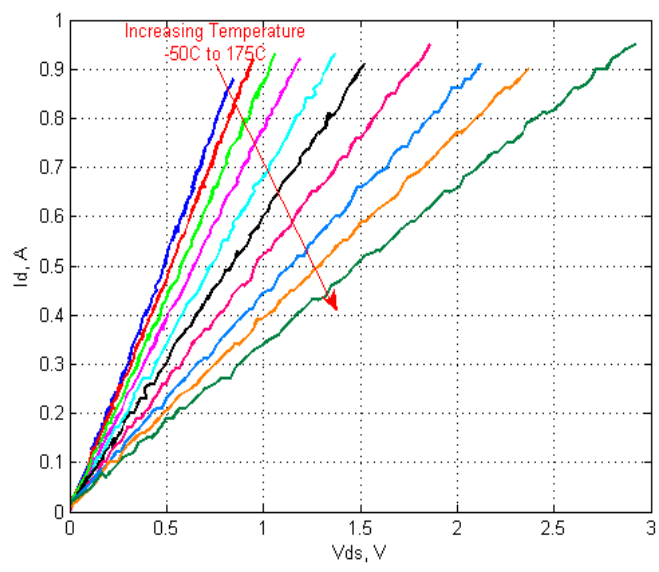

Fig. 10. $i-v$ characteristics of SiC VJFET at different temperatures.

and higher switching speed. JFET is free from the gate oxide interface problems unlike the MOSFET [9]. JFET is a normally-on device and conducts even though there is no gate voltage applied. Thus it requires protection circuit for system power failures to prevent a short circuit. This normally-on feature demands special gate drive designs increasing the complexity of design.

The VJFET can be used in high current and voltage applications, unlike Si JFET because of the vertical structure and the intrinsic properties of SiC. A normally-on SiC VJFET rated at $1200 \mathrm{~V}$ and $2 \mathrm{~A}$ was tested to study the high temperature behavior of the device. The forward characteristics for different temperatures are shown in Fig. 10. The on-resistance of the VJFET increases from 0.36 at $50^{\circ} \mathrm{C}$ to 1.4 at $175^{\circ} \mathrm{C}$ as shown in Fig. 11 . The values of the on-resistance are high; however, these devices have positive temperature coefficient which enables easy paralleling of devices and the on-state resistance would decrease.

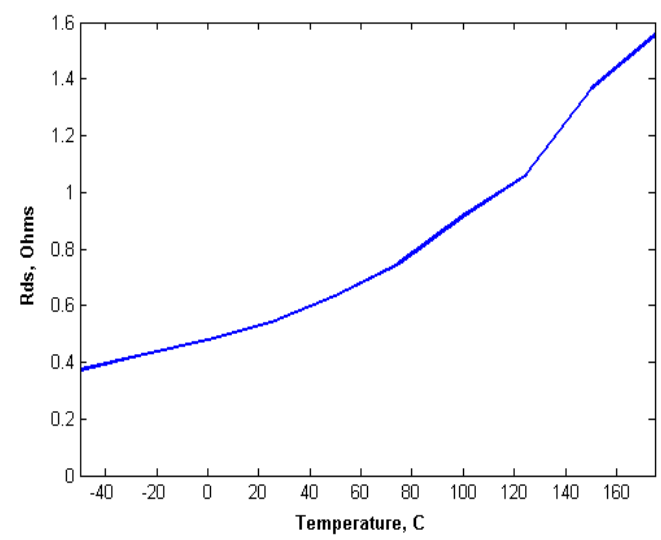

Fig. 11. On resistance of SiC VJFET at different temperatures.

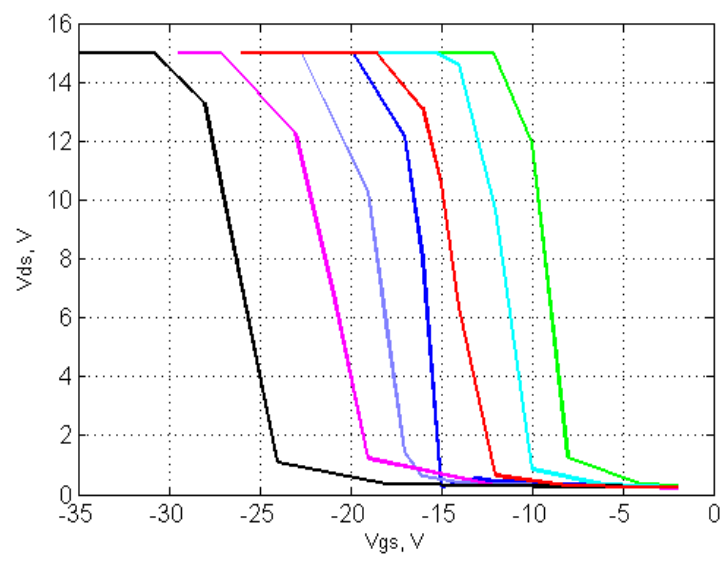

Fig. 12. Transfer characteristics of SiC VJFET.

\section{B. Transfer characteristics}

The transfer characteristics of different VJFET samples are shown in Fig. 12. The negative gate pinch-off voltage required to turn-off the device does not vary much with an increase in drain to source voltage $V_{d s}$. This pinch-off voltage determines the voltage requirement of the gate drive circuit.

A gate drive was developed for the VJFET for high frequency and high temperature operation. Based on the transfer characteristics, the gate drive was designed for a voltage of $-30 \mathrm{~V}$ and a $250 \mathrm{kHz}$ operation was achieved for peak gate currents of $0.8 \mathrm{~A}$.

\section{Gate drive requirements}

SiC VJFET switches can be operated at higher switching frequencies and higher temperatures; therefore, they have different gate drive requirements than traditional Si power switches. Since SiC VJFETs are normally-on devices they can be turned off by applying a negative voltage that is higher than what a typical Si switch requires.

One of the important parameters in gate drive design for VJFETs is that they have a large stray capacitance between the gate and the other terminals. Total input capacitance of VJFET, $C_{i s s}$, determines the current required by the gate and the rate at which the applied gate voltage is built across the gate and source terminals. These capacitances are due to the geometrical design of the device and also because of the parasitic body diode. Therefore, the circuit that drives the gate terminal should be capable of supplying a reasonable current so the stray capacitance can be charged up as quickly as possible.

The gate drive was tested with several capacitors as load before testing the driver circuit with the device. When these are operated at high frequencies, they also need high peak gate currents. The $C_{i s s}$ of VJFET is higher and a resistor is added between the gate driver and the source terminal. This is to limit the current to the gate so that the maximum voltage on the gate terminal does not overshoot. 
A SiC VJFET gate driver was built to satisfy these conditions and to utilize the most of the SiC device benefits. The main objective of the project was to build a gate driver using commercially available gate driver chips. Based on the transfer characteristics, the gate drive was designed for a voltage of $-30 \mathrm{~V}$ since the pinch-off voltage for most of the samples tested was $-20 \mathrm{~V}$. The gate series resistance was changed to achieve high frequency operation. A $250 \mathrm{kHz}$ operation was achieved with a resistance of $5.4 \mathrm{ohms}$ and with a peak gate current of 0.8A.

There are several gate drive circuits designed for $\mathrm{SiC}$ VJFETs using discrete devices [7], [8]. However, in the circuit design presented in this paper, a commercial gate drive IC chip IXDD414 is used which, makes it more reliable in operation. These gate drives can also be applied to $\mathrm{SiC}$ MOSFETs by redesigning the passive components and modifying the output voltage polarity. The gate voltage and current waveforms are shown in Fig. 13. The switching waveforms of the VJFET are shown in Fig. 14.

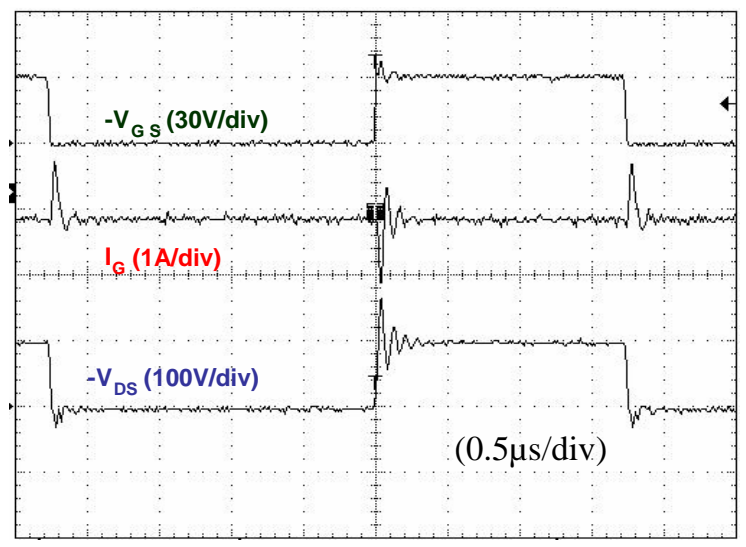

Fig. 13. Gate voltage and current waveforms at $250 \mathrm{kHz}$.

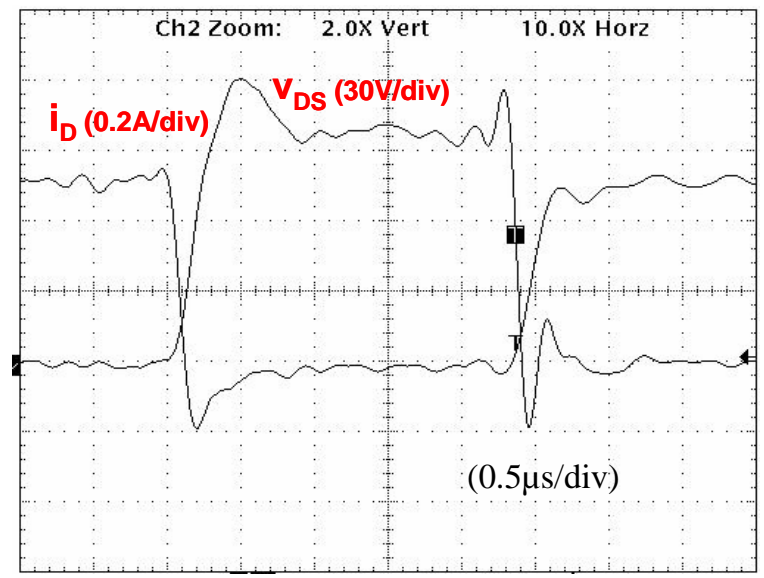

Fig. 14. Switching waveforms of SiC VJFET at $250 \mathrm{kHz}$.

\section{CONCLUSION}

The performances of some SiC Schottky diodes and a SiC JFET have been characterized for a wide temperature range. These characteristics will eventually be used to develop more realistic system level models to show the system level benefits of SiC devices.

SiC switching devices like the SiC JFET have different gate driver requirements. This paper, as opposed to the others in the literature, has identified a gate driver chip for MOSFETs and IGBTs and has shown how to modify it to drive SiC VJFETs. However, further research is required for higher temperature operation of these gate drivers.

\section{REFERENCES}

[1] B. Ozpineci, L. M. Tolbert, "Characterization of SiC Schottky diodes at different temperatures," IEEE Power Electronics Letters, vol. 1, no. 2, June 2003, pp. 54-57.

[2] B. Ozpineci, L. M. Tolbert, S. K. Islam, F. Z. Peng, “Testing, characterization, and modeling of SiC diodes for transportation applications," IEEE Power Electronics Specialists Conference, June 23-27, 2002, Cairns, Australia, pp. 1673-1678.

[3] L. M. Tolbert, B. Ozpineci, S. K. Islam F. Z. Peng, "Impact of SiC power electronic devices for hybrid electric vehicles," 2002 Future Car Congress Proceedings, June 3-5, 2002, Arlington, Virginia. (SAE paper number 2002-01-1904).

[4] B. Ozpineci, L. M. Tolbert, S. K. Islam, M. Hasanuzzaman, "Effects of silicon carbide (SiC) power devices on PWM inverter losses," The 27th Annual Conference of the IEEE Industrial Electronics Society, November 29 - December 2, 2002, Denver, Colorado, pp. 1061-1066.

[5] B. J. Baliga, "The future of power semiconductor device technology," Proceedings of the IEEE, vol. 89, no. 6, June 2001, pp. 822-832.

[6] A. R. Hefner, R. Singh, J. Lai, D. W. Berning, S. Bouche, C. Chapuy, "SiC power diodes provide breakthrough performance for a wide range of applications," IEEE Transactions on Power Electronics, vol. 16, no. 2, March 2001, pp. 273-280.

[7] K. Mino, K, S. Herold, J. W. Kolar, "A gate drive circuit for silicon carbide JFET," The 29th Annual Conference of the IEEE Industrial Electronics Society, vol. 2, 2-6 November 2003, pp. $1162-1166$.

[8] M. L. Heldwein, J. W. Kolar, “A novel SiC J-FET gate drive circuit for sparse matrix converter applications," Nineteenth Annual IEEE Applied Power Electronics Conference and Exposition, vol. 1, 22-26 February 2004, pp. 116-121.

[9] B. Allebrand, H. Nee, "On the possibility to use SiC JFETs in power electronic circuits," Proceedings of the $9^{\text {th }}$ European Conference on Power Electronics and Applications, Austria, 2001.

[10] M. Bhatnagar, P. K. McLarty, B. J. Baliga, "Silicon carbide high voltage $(400 \mathrm{~V})$ Schottky barrier diodes," IEEE Electron Device Letters, vol. 13, no. 10, October 1992, pp. 501-503. 